\title{
Monocytoid B-Lymphocyte
}

National Cancer Institute

\section{Source}

National Cancer Institute. Monocytoid B-Lymphocyte. NCI Thesaurus. Code C33138.

A lymphocyte derived most often from a pre-germinal center B cell, less often from a post-germinal center B cell. It is characterized by an abundant pale cytoplasm, indented nuclei with inconspicuous nucleoli, and open chromatin. It is found in the marginal zones of lymph nodes adjacent to the subcapsular and intermediary sinuses. 